\title{
SPECTRAL THEORY OF COMMUTING OPERATORS OF RANK TWO WITH PERIODIC COEFFICIENTS
}

\author{
S. P. NOVIKOV AND P. G. GRINEVICH
}

In the Novikov-Krichever formula for a fourth-order operator $L_{4}$, occurring in a commuting pair of pank two and genus one, there is an arithmetical error; the operator $L_{4}$ has the form

$$
L_{4}=\left(d^{2} / d x^{2}+u\right)^{2}+a d / d x+d / d x a+b
$$

(one of the terms $d / d x$ a has been omitted), where

$$
a=\frac{\lambda_{1}^{\prime}}{\mu_{1}}\left(\lambda_{1}-\lambda_{2}\right), \quad b=-\lambda_{1}-\lambda_{2}, \quad \lambda_{i}=\wp\left(\gamma_{i}\right), \quad \mu_{i}=-\wp^{\prime}\left(\gamma_{i}\right) .
$$

For all the quotations and formulas see the paper by Grinevich in this issue.

Theorem 1. The operator $L_{4}$ is formally symmetric if and only if $a=0$, i.e., $\lambda_{1}=\lambda_{2}$ (the constant $\gamma_{0}=0$ modulo the semiperiods of the function $\wp$ ).

In this case the formulas for the coefficients are strongly simplified:

$$
\begin{gathered}
a=0, \quad b=-2 \lambda, \text { where } \lambda=\wp(c(x)), \\
u=\frac{1}{4} \frac{\lambda^{\prime \prime 2}}{\lambda^{\prime 2}}-\frac{1}{2} \frac{\lambda^{\prime \prime \prime}}{\lambda^{\prime}}-\frac{1}{4} \frac{P_{3}(\lambda)}{\lambda^{\prime 2}} .
\end{gathered}
$$

Theorem 2. Assume that the following conditions hold: 1) the Riemann surface $\Gamma$ is real (i.e., $g_{1}, g_{2}$ are real); 2) the function $\lambda(x)$ is real; 3) at the points where $\lambda^{\prime}=0$ we have $\lambda^{\prime \prime} \neq 0, \lambda^{\prime \prime 2}=P_{3}(\lambda)$. Then, the operator $L_{4}$, defined by $(1),(2)$, has nonsingular real periodic coefficients and is a semibounded self-adjoint operator in $L_{2}(\mathbb{R})$.

For any linear ordinary differential operator $L$ (with respect to $x$ ) with periodic coefficients there is defined a "monodromy matrix" $\hat{T}(\lambda)$, i.e., a shift operator on the period of solutions of the equation $L \psi=\lambda \psi$, written in a certain basis. The order of the matrix $\hat{T}(\lambda)$ is equal to the order $k$ of the operator $L$. The eigenvectors $\psi_{q}(x, \lambda)$ of the operator $\hat{T}(\lambda)$ are called the Bloch eigenfunctions (or the Floquet functions). The function $\psi_{q}(x, \lambda)$ is meromorphic in $\lambda$ on the Riemann surface $\tilde{\Gamma}$ over the $\lambda$-plane of $k$ sheets, whose points are the pairs $Q_{q}=(\lambda, q), q=1, \ldots, k$.

Theorem 3. Assume that the operator $L$ of order $k=n l$ occurs in the commuting $\operatorname{pair}[A, L]=0$ of rank $l$ with Riemann surface $\Gamma$ of finite genus $\{P(A, L)=0\}$. Then, the monodromy matrix $\hat{T}(\lambda), \lambda \in \mathbb{C}$, written in the basis of the BakerAkhiezer eigenfunctions $\varphi_{\alpha}\left(x, \mathcal{P}_{i}\right), \alpha=1, \ldots$, , defines a matrix $\hat{T}^{*}(\mathcal{P})$ of order

Date: Original article submitted May 22, 1981.

UDC. 517.43 .

Translated from Funktsional'nyi Analiz i Ego Prilozheniya, Vol. 16, No. 1, pp. 25-26, JanuaryMarch, 1982. 
$l$ on the $n$-sheeted Riemann surface $\Gamma$ over the $\lambda$-plane, where $\mathcal{P}_{j}=(\lambda, j), j=$ $1, \ldots, n$. In this case the matrix $\hat{T}(\lambda)$ is in block form,

$$
\begin{gathered}
\hat{T}(\lambda)=\left(\begin{array}{ccc}
\hat{T}^{*}\left(\mathcal{P}_{1}\right) & & \\
& \ddots & \\
& & \hat{T}^{*}\left(\mathcal{P}_{n}\right)
\end{array}\right), \\
n=k / l, \quad \pi\left(\mathcal{P}_{j}\right)=\lambda, \quad \pi: \Gamma \rightarrow \mathbb{C}, \pi(\lambda, j)=\lambda, \\
\mathcal{P}_{1}=(\lambda, 1), \ldots, \mathcal{P}_{n}=(\lambda, n) .
\end{gathered}
$$

For operators of rank $l=2$ and order $k=n l=4$ the matrix $\hat{T}^{*}(\mathcal{P})$ on a twosheeted surface $\Gamma$ is unimodular. In the self-adjoint case the spectrum in $L_{2}(\mathbb{R})$ of the operator $L_{4}$ lies in the "real" subset $\Gamma_{\mathbb{R}} \subset \Gamma$, invariant relative to the complex conjugation (anti-involution) $\sigma: \Gamma \rightarrow \Gamma, \sigma\left(\Gamma_{\mathbb{R}}\right)=\Gamma_{\mathbb{R}}$, where the matrix $\hat{T}^{*}$ is real. For the genus $g=1$, the image of $\pi\left(\Gamma_{\mathbb{R}}\right)$ on the $\lambda$-line coincides with the set $P_{3}(\lambda)=4 \lambda^{3}+g_{2} \lambda+g_{3}>0$. In analogy with the usual second-order Schrödinger (Sturm-Liouville, Hill) operator, the spectrum is isolated in the set $\pi\left(\Gamma_{\mathbb{R}}\right)$ by the condition $\mathrm{Sp} \hat{T}^{*} \leq 2$.

Conclusions. Thus, the spectral theory of periodic operators of order $k=n l$ of rank $l$ is similar to the spectral theory of order $l$ over a Riemann surface instead of the $\lambda$-plane (the spectral parameter becomes "effectively" not rational but algebraic). Conversely, probably, if the monodromy matrix $\hat{T}^{*}(\lambda)$ reduces to the block form (3), where $\hat{T}^{*}(\mathcal{P})$ is defined on an algebraic $n$-sheeted Riemann surface $\Gamma$, then one has a non-trivial differential operator $A$ of some order $s=m l=m k / n$, which commutes with $L,[A, L]=0$. In this case the pair $(A, L)$ has $\operatorname{rank} l=k / n$. The converse statement has not been proved so far. The direct statement is proved similarly to the fundamental theorem of [1] for $l=1$ by the scheme presented in a more general, suitable and invariant form in the survey [2] (Chap. II).

Problem. Investigate the spectral properties of the operator $L$ in $L_{2}(\mathbb{R})$, if the coefficients are polynomial or rational for the rank $l>1$ (e.g., for the "Dixmier operator").

Conjecture. We fix the order $k$ only for one operator $L$ (we do not fix the order of $A$ ). Then the operators $L$ of $\operatorname{rank} l=1$ are everywhere dense among all periodic operators of order $k$.

The authors are grateful to I. M. Krichever for his help in the proof of the unimodularity of the matrix $\hat{T}^{*}$

\section{REFERENCES}

[1] S. P. Novikov, "The periodic problem for the Korteweg-de Vries equation. I," Funkts. Anal. Prilozhen., 8, No. 3, 54-66 (1974).

[2] B. A. Dubrovin, V. B. Matveev, and S. P. Novikov, "Nonlinear equations of Korteweg-de Vries type, finite-zone linear operators and Abelian varieties," Usp. Mat. Nauk, 31, No. 1, 55-136 (1976).

L. D. Landau Institute of Theoretical Physics, Academy of Sciences of the USSR. Moscow State University 